\title{
Post-Aneurysmal Subarachnoid Hemorrhage Vasospasm, Clinical Correlation between the Aneurysm Site and Clinical Vasospasm
}

\author{
Ahmed Ali, Mohamed A.R. Soliman \\ Neurosurgery Department, Faculty of Medicine, Cairo University, Cairo, Egypt \\ Email: moh.ar.sol@kasralainy.edu.eg
}

How to cite this paper: Ali, A. and Soliman, M.A.R. (2018) Post-Aneurysmal Subarachnoid Hemorrhage Vasospasm, Clinical Correlation between the Aneurysm Site and Clinical Vasospasm. Open Journal of Modern Neurosurgery, 8, 306-314. https://doi.org/10.4236/ojmn.2018.83025

Received: June 22, 2018

Accepted: July 21, 2018

Published: July 24, 2018

Copyright ( 92018 by authors and Scientific Research Publishing Inc. This work is licensed under the Creative Commons Attribution International License (CC BY 4.0).

http://creativecommons.org/licenses/by/4.0/

\begin{abstract}
Background: Intracranial vasospasm is a common complication following subarachnoid hemorrhage (SAH). The radiographic vasospasm can reach up to $90 \%$ of aneurysmal SAH. Materials and Methods: 139 consecutive patients admitted to Cairo University Hospitals from June 2013 to September 2014 with $\mathrm{SAH}$ who had been enrolled in a retrospective controlled study were analyzed retrospectively for the occurrence of vasospasm. The data collected from the charts of Cairo University Hospitals were the patient's demographics, clinical presentation, aneurysm location, treatment modality, and Glasgow Outcome Scale (GOS) scores. We excluded 24 patients with nonaneurysmal SAH, 3 internal carotid artery (ICA) aneurysms, 7 with multiple aneurysms and 4 patients died before treatment. Results: 72 males and 29 females were included in the study, mean age $53.5 \pm 11.5$ years. Twelve patients had aneurysms located in the vertebral artery group, 24 had middle cerebral artery aneurysms, 11 had pericallosal aneurysms, and 54 patients had anterior communicating artery (ACoA) complex aneurysms. Radiographic vasospasm occurred in $62.4 \%$ with the highest incidence $(75.9 \%)$ at the ACoA complex group. Symptomatic vasospasm occurred in $48.5 \%$ with the highest incidence $(63 \%)$ at the anterior communicating artery complex aneurysm location. The mean GOS at 6 months follow-up was 4.2. The worse GOS was found in the vertebral artery (VA) aneurysm group with a mean of 3.75. Conclusion: Aneurysms of the anterior communicating artery complex group have a greater risk of both radiographic and clinical vasospasm. Also, the worse 6 months follow-up GOS when an aneurysm was located in the VA group.
\end{abstract}

\section{Keywords}

Aneurysm Location, Subarachnoid Hemorrhage (SAH), Vasospasm, Glasgow Outcome Scale (GOS) 


\section{Introduction}

Intracranial vasospasm is a common complication following subarachnoid hemorrhage (SAH). The vasospasm can be either radiographic vasospasm (angiographic vasospasm) or it can be symptomatic vasospasm (Delayed Ischemic Neurologic Deficit (DIND)) [1]. The radiographic vasospasm can reach up to $90 \%$ of aneurysmal SAH [2].

In aneurysmal $\mathrm{SAH}$, two third of the cases will have a moderate vasospasm in at least one of the cerebral arteries. Half of these patients will develop symptoms due to ischemia [3]. Cerebral infarction is significantly associated with increasing patient age, history of diabetes mellitus, hypertension, chest infection, worse neurological status on admission, induced hypertension, and large aneurysms [4].

Symptoms of cerebral vasospasm most commonly appear between the $4^{\text {th }}$ and the $12^{\text {th }}$ day after the onset of SAH but can occur later; accordingly, it is important to remain cautious for this complication for at least 2 weeks after SAH. Symptomatic vasospasm usually has a gradual onset which can be heralded by behavioral changes or increased headache, so careful and regular bedside examination remains the most effective and simplest means of detecting early ischemia in examinable and awake patients; one should search for subtle findings such as decreased concentration, changes in verbal output, or newly developed pronator drift of the upper extremity. Only a small percentage of patients developed precipitous deterioration [5].

There are several causes of delayed deterioration after SAH that must be ruled out by radiological and laboratory investigations before diagnosing symptomatic vasospasm, these causes are; rebleeding of aneurysms or aneurysmal remnant, increased edema surrounding hematomas, or infarcts, hydrocephalus, hyponatremia, or infection [6].

We sought to study the relationship between the vasospasm (clinical and radiographic) and aneurysm location in patients enrolled in a retrospective controlled study.

\section{Materials and Methods}

139 consecutive patients admitted to Cairo University Hospitals from June 2013 to September 2014 with SAH who had been enrolled in a retrospective controlled study were analyzed retrospectively for the occurrence of vasospasm. The data collected from the charts of Cairo University Hospitals were the patient's demographics, clinical presentation, aneurysm location, treatment modality, and neurological outcomes (Glasgow Outcome Scale scores). We excluded 13 patients with nonaneurysmal SAH, 11 patients with Hunt and Hess Grade 4 and 5, 3 ICA aneurysms, 7 with multiple aneurysms and 4 patients died before treatment.

\subsection{Clinical Course}

All the patients were admitted to an intensive care unit and nimodipine was 
given to all patients. Ventricular drainage was done when necessary. Hypertensive therapy was administered when vasospasm is suspicious and continued after confirmation of vasospasm radiographic. All the patients were operated upon by microsurgical clipping with 48 - 72 hours from admission. All patients were assessed for vasospasm in the form of CT angiography or 4-vessel angiography on day 10 post-ictus or if there is any neurological alteration before day 10 . If there is clinical or radiological vasospasm, hypertensive therapy was administered. All the patients were treated by microsurgical clipping. All the patient were assessed clinically using Glasgow Outcome Scale [7] (Table 1) postoperatively daily till the day the day of discharge, one-week, one-month, three-month and 6 month post-discharge.

\subsection{Grouping of Aneurysms by Location}

Based on the aneurysm's proximity to cisterns or fissures the aneurysms were grouped into 1 of 4 locations. The first group was the vertebral artery (VA) location, which included patients with aneurysms of the intradural VA, basilar tip aneurysms, posterior communicating artery and the posterior inferior cerebellar artery (PICA). The second group included the middle cerebral artery (MCA) aneurysms. The third group included the pericallosal arteries aneurysms. The last group included the anterior communicating artery (ACoA) aneurysms, A1, and A2. All the results were compared in terms of mean value and percentage.

\subsection{Statistical Analysis}

Statistical analysis was performed using GraphPad Prism Software (GraphPad Inc.). For categorical variables, the Fisher exact test was used when comparing 2 groups with 2 outcome categories.

\section{Results}

One hundred and one patients were included in this study (72 males and 29 females, mean age $53.5 \pm 11.5$ years). Twelve patients had aneurysms located in the vertebral artery group, 24 had middle cerebral artery aneurysms, 11 had pericallosal aneurysms, and 54 patients had anterior communicating artery complex aneurysms (Table 2).

Table 1. Glasgow outcome scale [7].

\begin{tabular}{|c|c|c|}
\hline GOS & Functional status & Interpretation \\
\hline 1 & Death & Severe injury or death without recovery of consciousness \\
\hline 2 & Persistent vegetative state & $\begin{array}{l}\text { Severe damage with prolonged state of } \\
\text { unresponsiveness and a lack of higher mental functions }\end{array}$ \\
\hline 3 & Severe disability & Severe injury with permanent need for help with daily living \\
\hline 4 & Moderate disability & $\begin{array}{l}\text { No need for assistance in everyday life, } \\
\text { employment is possible but may require special equipment }\end{array}$ \\
\hline 5 & Low disability & $\begin{array}{l}\text { Light damage with minor neurological } \\
\text { and psychological deficits }\end{array}$ \\
\hline
\end{tabular}


Table 2. Distribution of the aneurysms according to the location.

\begin{tabular}{ccc}
\hline Group & $\begin{array}{c}\text { No. of Patients } \\
(\mathrm{n}=101)\end{array}$ & Location of an aneurysm \\
\hline Vertebral Artery & 12 & Vertebral artery and PICA \\
Middle cerebral artery & 24 & MCA \\
Pericallosal artery & 11 & Pericallosal arteries \\
$\begin{array}{c}\text { Anterior communicating } \\
\text { artery complex }\end{array}$ & 54 & Anterior communicating artery, $\mathrm{A}_{1}$ and $\mathrm{A}_{2}$ \\
\hline
\end{tabular}

PICA $=$ Posterior Inferior Cerebellar Artery, $A_{1}=$ first segment of anterior cerebral artery, $A_{2}=$ second segment of anterior cerebral artery, MCA = Middle Cerebral Artery.

\subsection{Vasospasm Incidence}

Sixty-Three (62.4\%) patients developed radiographic vasospasm (Table 3). The highest incidence of vasospasm (75.9\%) was at the anterior communicating artery complex location group while the lowest incidence (16.7\%) in the vertebral artery group (Figure 1).

Symptomatic vasospasm occurred in 49 patients (48.5\%). The highest incidence of symptomatic vasospasm (63\%) was the anterior communicating artery complex aneurysm location while the lowest incidence (8.3\%) was also the vertebral artery group (Figure 2).

\subsection{Outcomes}

The mean Glasgow Outcome Score (GOS) (Table 4) in 101 patients at 6 months follow-up was 4.2. The worse GOS was found in the VA aneurysm group with a mean of 3.75 and the best GOS was found in the MCA group with a mean score of 4.5.

We divided the outcome into two groups: Poor outcome (GOS 1 - 3) and good outcome (GOS 4 - 5). There was a poor outcome in $26(25.7 \%)$ of 101 patients. There were good outcomes (GOS 4 - 5) in 75 (74.3\%) of 101 patients. (Figure 3)

The highest relative risk for symptomatic vasospasm was found in the anterior communicating artery complex location group with a ratio of 1.97 and the lowest relative risk was found in the vertebral artery group with a ratio of 0.15 (Table 5).

\section{Discussion}

The greatest cause of morbidity in SAH patients is the cerebral vasospasm causing delayed ischemia [1] [8] [9]. An important prognosticator in the SAH patients is to identify the patient who at a greater risk of developing cerebral and who will likely need prompt management like vasospasm endovascular treatment and/or hypertensive therapy. Harrod and colleagues wrote a systematic review of studies from 1996 to 2005 and the review showed that the burden of blood at the time of SAH is the only predictive factor of cerebral vasospasm [1]. 


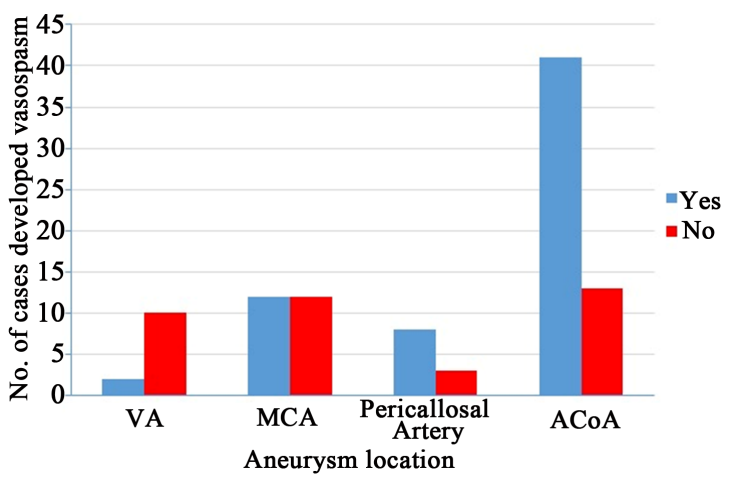

Figure 1. Bar chart showing the presence of any vasospasm in each of the 4 aneurysm location groups. $\mathrm{VA}=$ Vertebral Artery, $\mathrm{ACoA}_{1}=$ Anterior Communicating Artery Aneurysm MCA = Middle Cerebral Artery.

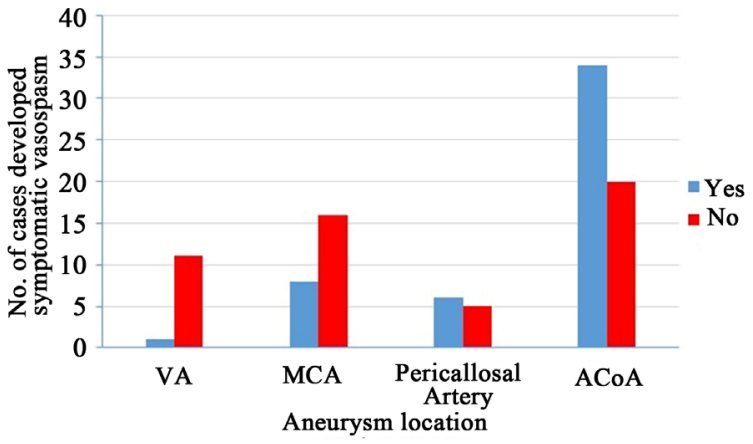

Figure 2. Bar chart showing the presence of symptomatic vasospasm in each of the 4 aneurysm location groups. VA $=$ Vertebral Artery, ACoA1 = Anterior Communicating Artery Aneurysm MCA = Middle Cerebral Artery.

Table 3. The rate of vasospasm according to location.

\begin{tabular}{cccccc}
\hline Variable & Overall & VA & MCA & Pericallosal & ACoA \\
\hline $\begin{array}{c}\text { No. of patients } \\
\text { Symptomatic vasospasm }\end{array}$ & 101 & 12 & 24 & 11 & 54 \\
Yes & $49(48.5 \%)$ & $1(8.3 \%)$ & $8(33.3 \%)$ & $6(54.5 \%)$ & $34(63 \%)$ \\
No & $52(51.5 \%$ & $11(91.7 \%)$ & $16(66.7 \%)$ & $5(45.5 \%)$ & $20(37 \%)$ \\
Radiographic vasospasm & & & & & \\
Yes & $63(62.4 \%)$ & $2(16.7 \%)$ & $12(50 \%)$ & $8(72.7 \%)$ & $41(75.9 \%)$ \\
No & $38(37.6 \%)$ & $10(83.3 \%)$ & $12(50 \%)$ & $3(27.3 \%)$ & $13(24.1 \%)$ \\
\hline
\end{tabular}

$\mathrm{VA}=$ Vertebral Artery, $\mathrm{ACoA}_{1}=$ Anterior Communicating Artery Aneurysm MCA $=$ Middle Cerebral Artery.

Table 4. GOS by aneurysm location.

\begin{tabular}{ccccc}
\hline GOS & VA $(\mathrm{n}=12)$ & MCA $(\mathrm{n}=24)$ & Pericallosal Arteries $(\mathrm{n}=11)$ & ACoA $(\mathrm{n}=54)$ \\
\hline 1 & 2 & 2 & 1 & 9 \\
2 & 0 & 0 & 0 & 0 \\
3 & 2 & 1 & 0 & 3 \\
4 & 3 & 2 & 5 & 4 \\
5 & 5 & 19 & 5 & 38 \\
\hline
\end{tabular}

$\mathrm{VA}=$ Vertebral Artery, $\mathrm{ACoA}_{1}=$ Anterior Communicating Artery Aneurysm MCA $=$ Middle Cerebral Artery. 


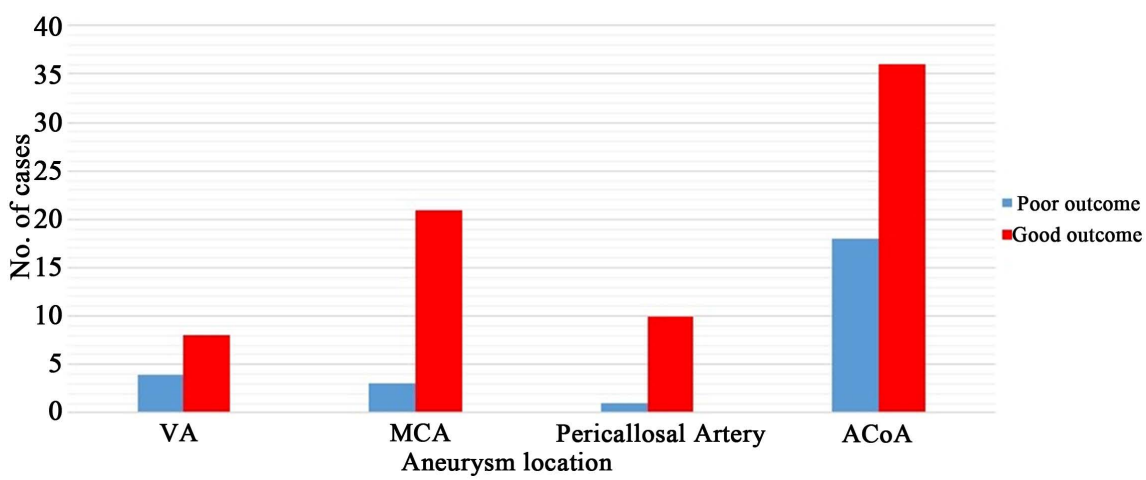

Figure 3. Graph of GOS at 6 months follow-up in each of the 4 aneurysms locations groups showing either poor outcome (GOS 1 - 3) or good outcome (GOS 4 - 5). VA = Vertebral Artery, $\mathrm{ACoA}_{1}=$ Anterior Communicating Artery Aneurysm MCA $=$ Middle Cerebral Artery.

Table 5. Symptomatic vasospasm risk by aneurysm location.

\begin{tabular}{ccc}
\hline Aneurysm Location & Relative risk ratio & Odd Ratio \\
\hline VA & 0.15 & 0.08 \\
MCA & 0.63 & 0.44 \\
Pericallosal Artery & 1.14 & 1.31 \\
ACoA & 1.97 & 3.63 \\
\hline
\end{tabular}

$\mathrm{VA}=$ Vertebral Artery, $\mathrm{ACoA}_{1}=$ Anterior Communicating Artery Aneurysm MCA $=$ Middle Cerebral Artery.

Kistler and colleagues investigated the relation of the location of blood and cerebral vasospasm in 41 patients [8]. They found that the arterial territory involved in the vasospasm was related to the site of the hemorrhage [8]. These results were similar to some previous studies, which correlated the aneurysm location with the cerebral infarction territory [10]. However, the study population was only 41 patients, 20 patients only experienced vasospasm [8].

Yin and colleagues studied 211 patients retrospectively and stratified the aneurysm location into four groups: no aneurysm, posterior circulation, anterior circulation, or unclear. In this study they didn't mention the relation between vasospasm and aneurysm location, may be due to a large number of aneurysms locations [9].

Abla and colleagues studied 216 patients with treated ruptured aneurysm in a randomized controlled trial and stratified the aneurysm location into six groups: intradural ICA, vertebral artery, basilar artery, pericallosal artery, middle cerebral artery, and anterior communicating artery complex. They investigated the relationship between vasospasm and ruptured aneurysm location. Their results showed that there is no significant difference between aneurysm location and the rate of clinical and radiological vasospasm except in the pericallosal artery group had the highest risk of vasospasm. They also analyzed the outcome of the 6 aneurysms location groups and concluded that the worst mean 1-year modified 
Rankin scale (mRS) score was in the lower VA aneurysms patients [11].

In our series of 129 patients in a retrospective controlled study, we analyzed retrospectively the relationship between vasospasm and the location of the ruptured aneurysm. Our results showed that when we grouped aneurysms according to location into 4 different groups, the highest incidence of radiographic vasospasm $(75.9 \%)$ was at the anterior communicating artery complex location group, followed by the pericallosal artery group with an incidence of $72.7 \%$ followed by the middle cerebral artery group with an incidence of $50 \%$ and the lowest incidence (16.7\%) was in the vertebral artery group.

Not all the patients with radiological vasospasm developed symptoms of clinical vasospasm. The anterior communicating artery complex location group remained the highest incidence in the patients with symptomatic vasospasm with an incidence of $63 \%$ followed by the pericallosal artery group with an incidence of $54.5 \%$ followed by the middle cerebral artery group (33.3\%) and the lowest incidence $(8.3 \%)$ was the vertebral artery group.

We think that the main cause for the increased incidence of vasospasm among the anterior communicating artery complex location group aneurysms is the complexity of the location with very important structures and many perforators supply critical structures.

We also analyzed the outcome of the patients in the 4 aneurysms location groups by using the Glasgow Outcome Score (GOS) in 6-months follow-up. The best prognosis was in the middle cerebral artery group with a mean GOS 6-months follow-up of 4.5 followed by the pericallosal artery group with a mean GOS 6-months follow-up of 4.18 followed by the anterior communicating artery complex location group with a mean GOS 6-months follow up of 3.8 and the worst prognosis was the vertebral artery group with a mean GOS 6-months follow-up of 3.75.

The vertebral artery group has the worst prognosis may be due to the proximity to the lower cranial nerves which might lead to difficulty in swallowing, and ventilator dependency. The anterior communicating artery complex had the second worst prognosis may be due to the complexity of this location with perforators and critical structures.

This study has some limitations because it is a retrospective study. Furthermore, 24 non-aneurysmal SAH patients and 4 patients died before treatment was excluded from this study. All the patients were treated by microvascular clipping with no comparison between the coiling and clipping effect on the outcome of these patients.

\section{Conclusion}

In this study, we found that aneurysms of the anterior communicating artery complex location group have a greater risk of both radiographic and clinical vasospasm. Also, the patients in this study have the worse 6 months follow-up GOS outcome when an aneurysm was located in the VA group (including the PICA). 


\section{References}

[1] Harrod, C.G., Bendok, B.R. and Batjer, H.H. (2005) Prediction of Cerebral Vasospasm in Patients Presenting with Aneurysmal Subarachnoid Hemorrhage: A Review. Neurosurgery, 56, 633-654. https://doi.org/10.1227/01.NEU.0000156644.45384.92

[2] Dorsch, N.W.C. and King, M.T. (1994) A Review of Cerebral Vasospasm in Aneurysmal Subarachnoid Haemorrhage: I. Incidence and Effects. Journal of Clinical Neuroscience, 1, 19-26. https://doi.org/10.1016/0967-5868(94)90005-1

[3] Fergusen, S. and MacDonald, R.L. (2007) Predictors of Cerebral Infarction in Patients with Aneurysmal Subarachnoid Hemorrhage. Neurosurgery, 60, 658-667. https://doi.org/10.1227/01.NEU.0000255396.23280.31

[4] Proust, F., Hannequin, D., Langlois, O., Freger, P. and Crissard, P. (1995) Causes of Morbidity and Mortality after Ruptured Aneurysm Surgery in a Series of $230 \mathrm{~Pa}$ tients: The Importance of Control Angiography. Stroke, 26, 1553-1557.

https://doi.org/10.1161/01.STR.26.9.1553

[5] Fisher, C.M., Roberson, G.H. and Ojemann, R.G. (1977) Cerebral Vasospasm with Ruptured Saccular Aneurysm-The Clinical Manifestations. Neurosurgery, 1, 245-258. https://doi.org/10.1227/00006123-197711000-00004

[6] Kramer, A.H., Hehir, M., Nathan, B. and Gress, D. (2008) A Comparison of 3 Radiographic Scales for the Prediction of Delayed Ischemia and Prognosis Following Subarachnoid Hemorrhage. Journal of Neurosurgery, 109, 199-207. https://doi.org/10.3171/JNS/2008/109/8/0199

[7] Jennett, B. and Bond, M. (1975) Assessment of Outcome after Severe Brain Damage. Lancet, 1, 480-484. https://doi.org/10.1016/S0140-6736(75)92830-5

[8] Kistler, J.P., Crowell, R.M., Davis, K.R., Heros, R., Ojemann, R.G., Zervas, T., et al. (1983) The Relation of Cerebral Vasospasm to the Extent and Location of Subarachnoid Blood Visualized by CT Scan: A Prospective Study. Neurology, 33, 424-436. https://doi.org/10.1212/WNL.33.4.424

[9] Yin, L., Ma, C.Y., Li, Z.K., Wang, D.D. and Bai, C.M. (2011) Predictors Analysis of Symptomatic Cerebral Vasospasm after Subarachnoid Hemorrhage. Acta Neurochirurgica Supplement, 110, 175-178.

[10] Rabinstein, A.A., Friedman, J.A., Weigand, S.D., McClelland, R.L., Fulgham, J.R., Manno, E.M., et al. (2004) Predictors of Cerebral Infarction in Aneurysmal Subarachnoid Hemorrhage. Stroke, 35, 1862-1866. https://doi.org/10.1161/01.STR.0000133132.76983.8e

[11] Abla, A., Wilson, D., Williamson, R., Nakaii, P., McDougall, C.G., Zabramski, J.M., et al. (2014) The Relationship between Ruptured Aneurysm Location, Subarachnoid Hemorrhage Clot Thickness, and Incidence of Radiographic or Symptomatic Vasospasm in Patients Enrolled in a Prospective Randomized Controlled Trial. Journal of Neurosurgery, 120, 391-397. https://doi.org/10.3171/2013.10.JNS13419 


\section{List of Abreviations}

$A_{1}: 1^{\text {st }}$ part of anterior cerebral artery

$\mathrm{A}_{2}: 2^{\text {nd }}$ part of anterior cerebral artery

ACoA: Anterior communicating artery

DIND: Delayed Ischemic Neurologic Deficit

GOS: Glasgow Outcome Score

ICA: Internal carotid artery

MCA: Middle cerebral artery

mRS: Modified Rankin scale

PICA: Posterior inferior cerebellar artery

SAH: Subarachnoid hemorrhage 